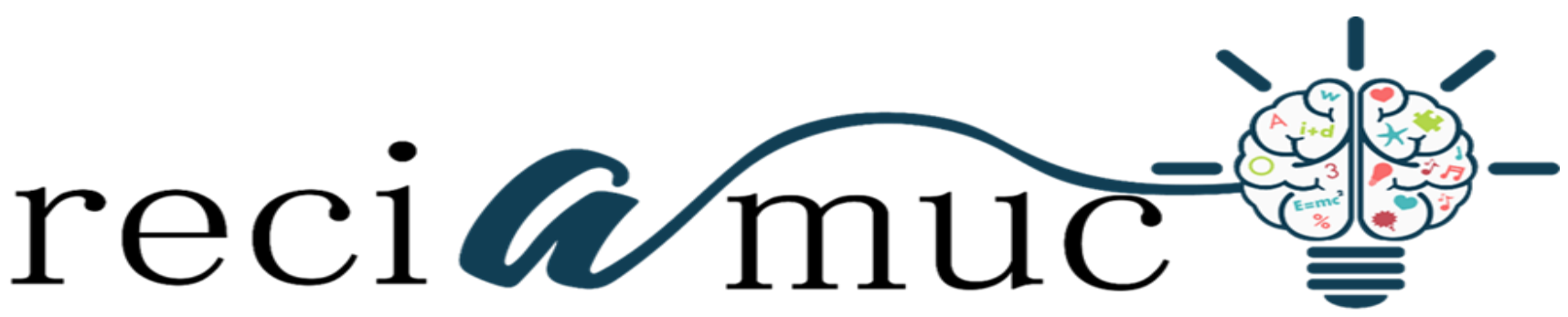

Revista cientifica de investigación actualización del mundo de las ciencias

Daniel Fernando Dick Paredes ${ }^{\text {a; }}$ Jenniffer Cristina Pazmiño Farfán ${ }^{\text {b; }}$ John Michael

Franco Orellana ${ }^{c}$; Adrián André Bravo Legarda ${ }^{d}$

Deshidratación en niños: Causas, diagnóstico y tratamiento

Dehydration in children: Therapies and prevention

Revista Científica de Investigación actualización del mundo de las Ciencias. Vol. 3 núm., 1, enero, ISSN: 2588-0748, 2018, pp. 181-198

DOI: $10.26820 /$ reciamuc/3.(1).enero.2019.181-198

URL: $\underline{\text { http://reciamuc.com/index.php/RECIAMUC/article/view/230 }}$

Código UNESCO: 3205 Medicina Interna

Tipo de Investigación: Artículo de Revisión

Editorial Saberes del Conocimiento

Recibido: 10/12/2018

Aceptado: 20/01/2019

Publicado: 30/01/2019

Correspondencia: director@recimauc.com

a. Médico; Guayaquil, Ecuador.
b. Médico; Guayaquil, Ecuador.
c. Médico; Guayaquil, Ecuador.
d. Médico; Guayaquil, Ecuador. 


\section{Deshidratación en niños: Causas, diagnóstico y tratamiento \\ Vol. 3, núm. 1., (2019) \\ Daniel Fernando Dick Paredes; Jenniffer Cristina Pazmiño Farfán; John Michael Franco Orellana; Adrián André Bravo Legarda}

\section{RESUMEN}

La deshidratación y sus complicaciones siguen siendo una causa importante de morbilidad y mortalidad en los niños, especialmente en los países en desarrollo. Una de las causas principales de la deshidratación es la diarrea la cual se caracteriza por un aumento de la frecuencia y el volumen, y una disminución de la consistencia de las heces dentro de los estándares normales. Los patógenos varían entre los distintos escenarios mundiales en cuanto a desarrollo. La diarrea por rotavirus es el agente etiológico más importante implicado en la deshidratación grave por diarrea. Si bien es importante reconocer la causa microbiológica específica de la diarrea para aplicar el tratamiento adecuado, los aspectos preventivos más amplios presentados por la Organización Mundial de la Salud (OMS) indican los contribuyentes fundamentales a la carga masiva de enfermedad en los países en desarrollo. El manejo de un niño con diarrea aguda debe incluir una historia y un examen exhaustivo con evaluación del estado de hidratación, estado nutricional y evaluación clínica integral para cualquier complicación o enfermedad asociada. Los avances más recientes en el área de la enfermedad diarreica aguda incluyen la administración de suplementos de zinc y la solución de rehidratación oral (SRO) con osmolaridad reducida y vacunación contra el rotavirus.

Palabras Claves: Deshidratación Infantil; Diarrea; Tratamiento; Prevención. 


\title{
Deshidratación en niños: Causas, diagnóstico y tratamiento
}

Vol. 3, núm. 1., (2019)

Daniel Fernando Dick Paredes; Jenniffer Cristina Pazmiño Farfán; John Michael Franco

Orellana; Adrián André Bravo Legarda

\begin{abstract}
Diarrheal diseases and their complications continue to be a major cause of morbidity and mortality in children, especially in developing countries. Diarrhea is characterized by an increase in frequency and volume, and a decrease in stool consistency within normal standards. Pathogens vary among different world scenarios in terms of development. Rotavirus diarrhea is the most important etiologic agent involved in severe dehydration due to diarrhea. Although it is important to recognize the specific microbiological cause of diarrhea to apply the appropriate treatment, the broader preventive aspects presented by the World Health Organization (WHO) indicate the fundamental contributors to the massive burden of disease in developing countries. The management of a child with acute diarrhea should include a history and a thorough examination with evaluation of hydration status, nutritional status and comprehensive clinical evaluation for any associated complication or disease. The most recent advances in the area of acute diarrheal disease include zinc supplementation and oral rehydration solution (ORS) with reduced osmolarity and vaccination against rotavirus.
\end{abstract}

Key Words: Infant Dehydration; Diarrhea; Treatment; Prevention. 


\section{Deshidratación en niños: Causas, diagnóstico y tratamiento \\ Vol. 3, núm. 1., (2019) \\ Daniel Fernando Dick Paredes; Jenniffer Cristina Pazmiño Farfán; John Michael Franco Orellana; Adrián André Bravo Legarda}

\section{Introducción.}

La deshidratación y sus complicaciones siguen siendo una causa importante de morbilidad y mortalidad infantil, especialmente en países en vías de desarrollo. Es la segunda causa más común de muerte en niños menores de cinco años de edad en todo el mundo y es responsable de 2,4 millones de muertes cada año (Forsberg \& Petzold, 2007). Informe de la carga de la enfermedad del Consejo de Investigación Médica indica que en Sudáfrica, es la tercera mayor asesina de niños menores de cinco años, responsables de más de 10000 muertes anuales (el 10,2\% del total de muertes) (Burden of Disease report, 2010) hay aproximadamente 1,5 billones de episodios de diarrea por año por lo que el conocimiento de la etiología y la gestión adecuada es esencial para todos los profesionales de la salud.

Los avances más recientes en el área de la enfermedad diarreica aguda incluyen suplementos de zinc, solución de rehidratación oral (ORS) de osmolaridad reducida y vacunación de rotavirus.

La diarrea se caracteriza por un aumento de la frecuencia y el volumen, y disminución de la consistencia de las heces de la norma. Cabe destacar que la frecuencia de evacuación de las heces varía con la edad y es mayor en bebés (Guarino \& Albano, 2008) La disentería se define como el paso de sangre y moco en las heces diarreicas. La diarrea persistente ocurre cuando la duración de los síntomas supera los siete días y la diarrea crónica cuando dura más de 14 días.

La incidencia de estos patógenos varía entre los entornos mundiales desarrollados y en vias de desarrollo. En los países desarrollados alrededor del 70\% de los casos de diarrea son virales ( $40 \%$ de rotavirus), 10 a 20\% de bacterias y <10\% de origen protozoario, en países en 


\section{Deshidratación en niños: Causas, diagnóstico y tratamiento}

Vol. 3, núm. 1., (2019)

Daniel Fernando Dick Paredes; Jenniffer Cristina Pazmiño Farfán; John Michael Franco

Orellana; Adrián André Bravo Legarda

vías de desarrollo 50-60\% de los casos son de bacterias (E. coli enteropatógena 25\%, Campylobacter jejuni 10-18\%, Shigella spp y Salmonella spp 5\% cada una), 35\% de origen viral (15 a 25\% de rotavirus), y en muchos casos la causa no está identificada o mixto. (Guarino \& Albano, 2008) En países en vías de desarrollo la prevalencia de diarrea también varía ampliamente según el país. Por ejemplo, hay muchos más casos de cólera en la India y el sudeste asiático, mientras que en África se ha demostrado que el rotavirus es el agente causante en 2849\% de casos en Etiopía, pero solo el 14\% de los casos en Tanzania (Naghipour, Nakgomi, \& Nakagomi, 2008). La incidencia

La diarrea por rotavirus varía ampliamente incluso en cada país con estudios de Sudáfrica que indican un rango de 14 a 34\% de los casos en Johannesburgo, 20-55\% en Durban y 18\% en Ciudad del Cabo. También hay una marcada estacionalidad que se asocia con la incidencia de diarrea infantil infecciosa. Esto se refleja mejor en la infección por rotavirus, descrita clásicamente en los meses secos de invierno en climas templados. (Naghipour, Nakgomi, \& Nakagomi, 2008). Esto está documentado en estudios de Durban y Johannesburgo, pero no en el pico de verano de la diarrea en Ciudad del Cabo y otros países en desarrollo. Este patrón es muy probable debido al clima mediterráneo en Ciudad del Cabo con inviernos húmedos y veranos calurosos y secos. Patógenos bacterianos como Campylobacter jejuni y Salmonella spp invade el revestimiento del intestino delgado y grueso por lo que los niños tienden a tener fiebres más altas. Estos organismos pueden diseminarse sistémicamente especialmente en niños pequeños. Las infecciones por Shigella dysenteriae y ETEC también pueden ser complicadas por el síndrome hemolítico urémico. Ciertos organismos están más estrechamente asociados con la diarrea crónica, y estos incluyen Giardia lamblia y Cryptosporidium parvum. 


\section{Deshidratación en niños: Causas, diagnóstico y tratamiento}

Vol. 3, núm. 1., (2019)

Daniel Fernando Dick Paredes; Jenniffer Cristina Pazmiño Farfán; John Michael Franco Orellana; Adrián André Bravo Legarda

La diarrea por rotavirus es el agente etiológico más importante implicado en todo el mundo en diarrea grave deshidratante que requiere hospitalización. La carga anual de enfermedad se estima como más de 110 millones de episodios de diarrea, 25 millones de visitas clínicas, 2 millones hospitalizaciones y 600000 muertes infantiles al año. Más del 90\% de las muertes por rotavirus ocurren en países en desarrollo. Las mejoras en el suministro de agua y saneamiento han demostrado reducir la transmisión de bacterias y parásitos entéricos, pero esto parece no tener un impacto importante en la diarrea por rotavirus, por lo que la introducción de una vacuna muestra la mayor promesa para reducir la carga de la enfermedad. La edad máxima de la infección se cita entre seis meses a dos años. En los países en desarrollo, sin embargo, los niños a menudo se presentan más jóvenes, con la edad media de hospitalización de la diarrea por todas las causas es de nueve meses en comparación con la de seis meses en la diarrea por rotavirus, con el 97\% de los casos ocurriendo en niños menores de 18 meses.

Algunos estudios indican que hasta el $38 \%$ de los pacientes con diarrea por rotavirus tenían menos de 6 meses de edad. Se requiere una pequeña dosis infecciosa $(<100$ partículas de virus) para entrar en el epitelio del intestino delgado donde elabora una potente enterotoxina que daña las células epiteliales causando embotamiento, vellosidades y desprendimiento viral masivo. Esto se traduce en una profusa diarrea acuosa no inflamatoria, deshidratación rápida y perdida de electrolitos, a menudo se asocia con fiebre inicial y vómitos durante dos o tres días, y el curso de la infección dura de dos a siete días.

El abordaje etiológico en la diarrea nosocomial también diferirá y hay que tener en cuenta la infección por Clostridium difficile. Esta es una espora formando anaerobios, y pacientes colonizados con una toxina productora. La cepa puede desarrollar una enfermedad especialmente 


\section{Deshidratación en niños: Causas, diagnóstico y tratamiento}

Vol. 3, núm. 1., (2019)

Daniel Fernando Dick Paredes; Jenniffer Cristina Pazmiño Farfán; John Michael Franco

Orellana; Adrián André Bravo Legarda

cuando se trata con antibióticos. Rotavirus y astrovirus son otras causas importantes de brotes de diarrea nosocomial a través de la transmisión fecal-oral y contaminación de superficies ambientales. Aunque es importante reconocer la especificidad microbiológica de las causas de la diarrea para tratar el tratamiento adecuado.

Los aspectos preventivos más amplios presentados por la OMS indican que los contribuyentes fundamentales a la carga masiva de la enfermedad en países en desarrollo en un entorno global donde hasta un cuarto de los niños están desnutridos, más de mil millones de personas no tienen acceso a agua potable y más de dos mil millones tienen un saneamiento inadecuado, juntos con una baja prevalencia de lactancia materna, desorganización social de la guerra y desastres naturales, así como la mala educación materna, los viciosos ciclos de infección de diarrea y desnutrición se perpetúa.

\section{Metodología.}

Para el desarrollo de este proceso investigativo, se plantea como metodología la encaminada hacia una orientación científica particular que se encuentra determinada por la necesidad de indagar en forma precisa y coherente una situación, en tal sentido (Davila, 2015) define la metodología "como aquellos pasos previos que son seleccionados por el investigador para lograr resultados favorables que le ayuden a plantear nuevas ideas".(p.66) 


\section{Deshidratación en niños: Causas, diagnóstico y tratamiento}

Vol. 3, núm. 1., (2019)

Daniel Fernando Dick Paredes; Jenniffer Cristina Pazmiño Farfán; John Michael Franco Orellana; Adrián André Bravo Legarda

Lo citado por el autor, lleva a entender que el desarrollo de la acción investigativa busca simplemente coordinar acciones enmarcadas en una revisión bibliográfica con el fin de complementar ideas previas relacionadas a la prevención de la deshidratación causada por diarrea en niños a través de una revisión de literatura, para así finalmente elaborar un cuerpo de consideraciones generales que ayuden a ampliar el interés propuesto.

\section{Tipo de Investigación}

Dentro de toda práctica investigativa, se precisan acciones de carácter metodológico mediante las cuales, se logra conocer y proyectar los eventos posibles que la determinan, así como las características que hacen del acto científico un proceso interactivo ajustado a una realidad posible de ser interpretada. En este sentido, se puede decir, que la presente investigación corresponde al tipo documental, definido por Castro (2016), "se ocupa del estudio de problemas planteados a nivel teórico, la información requerida para abordarlos se encuentra básicamente en materiales impresos, audiovisuales y /o electrónicos”. (p.41).

En consideración a esta definición, la orientación metodológica permitió la oportunidad de cumplir con una serie de actividades inherentes a la revisión y lectura de diversos documentos donde se encontraron ideas explicitas relacionadas con los tópicos encargados de identificar a cada característica insertada en el estudio. Por lo tanto, se realizaron continuas interpretaciones con el claro propósito de revisar aquellas apreciaciones o investigaciones propuestas por diferentes investigadores relacionadas con el tema de interés, para luego dar la respectiva argumentación a los planteamientos, en función a las necesidades encontradas en la indagación. 


\section{Deshidratación en niños: Causas, diagnóstico y tratamiento}

Vol. 3, núm. 1., (2019)

Daniel Fernando Dick Paredes; Jenniffer Cristina Pazmiño Farfán; John Michael Franco

Orellana; Adrián André Bravo Legarda

\section{Fuentes Documentales}

El análisis correspondiente a las características que predomina en el tema seleccionado, llevan a incluir diferentes fuentes documentales encargadas de darle el respectivo apoyo y en ese sentido cumplir con la valoración de los hechos a fin de generar nuevos criterios que sirven de referencia a otros procesos investigativos. Para (CASTRO, 2016) las fuentes documentales incorporadas en la investigación documental o bibliográfica, "representa la suma de materiales sistemáticos que son revisados en forma rigurosa y profunda para llegar a un análisis del fenómeno". (p.41). Por lo tanto, se procedió a cumplir con la realización de una lectura previa determinada para encontrar aquellos aspectos estrechamente vinculados con el tema, con el fin de explicar mediante un desarrollo las respectivas apreciaciones generales de importancia.

\section{Técnicas para la Recolección de la Información}

La conducción de la investigación para ser realizada en función a las particularidades que determinan a los estudios documentales, tiene como fin el desarrollo de un conjunto de acciones encargadas de llevar a la selección de técnicas estrechamente vinculadas con las características del estudio. En tal sentido, (Bolívar, 2015), refiere, que es "una técnica particular para aportar ayuda a los procedimientos de selección de las ideas primarias y secundarias”. (p. 71).

Por ello, se procedió a la utilización del subrayado, resúmenes, fichaje, como parte básica para la revisión y selección de los documentos que presentan el contenido teórico. Es decir, que mediante la aplicación de estas técnicas se pudo llegar a recoger informaciones en cuanto a la revisión bibliográfica de los diversos elementos encargados de orientar el proceso de investigación. Tal como lo expresa, (Bolívar, 2015) "las técnicas documentales proporcionan las 


\section{Deshidratación en niños: Causas, diagnóstico y tratamiento}

Vol. 3, núm. 1., (2019)

Daniel Fernando Dick Paredes; Jenniffer Cristina Pazmiño Farfán; John Michael Franco Orellana; Adrián André Bravo Legarda

herramientas esenciales y determinantes para responder a los objetivos formulados y llegar a resultados efectivos" (p. 58). Es decir, para responder con eficiencia a las necesidades investigativas, se introdujeron como técnica de recolección el método inductivo, que hizo posible llevar a cabo una valoración de los hechos de forma particular para llegar a la explicación desde una visión general.

Asimismo, se emplearon las técnicas de análisis de información para la realización de la investigación que fue ejecutada bajo la dinámica de aplicar diversos elementos encargados de determinar el camino a recorrer por el estudio, según, (Bolívar, 2015) las técnicas de procesamiento de datos en los estudios documentales "son las encargadas de ofrecer al investigador la visión o pasos que debe cumplir durante su ejercicio, cada una de ellas debe estar en correspondencia con el nivel a emplear" (p. 123). Esto indica, que para llevar a cabo el procesamiento de los datos obtenidos una vez aplicado las técnicas seleccionadas, tales como: fichas de resumen, textual, registros descriptivos entre otros, los mismos se deben ajustar al nivel que ha sido seleccionado.

\section{Resultados.}

El manejo de un niño que presenta diarrea aguda debe incluir una historia completa y un examen con evaluación de hidratación, estado nutricional y evaluación clínica integral para cualquier complicación o enfermedad asociada. Una decisión entonces se debe tomar en el método de rehidratación, alimentación, y si hay otras indicaciones para cualquier investigación farmacológica especializada la terapia usualmente se limita al soporte de micronutrientes. 


\section{Deshidratación en niños: Causas, diagnóstico y tratamiento}

Vol. 3, núm. 1., (2019)

Daniel Fernando Dick Paredes; Jenniffer Cristina Pazmiño Farfán; John Michael Franco

Orellana; Adrián André Bravo Legarda

La evaluación clínica después de obtener un historial de diarrea con o sin vómitos, es la primera prioridad en la evaluación inicial para identificar y tratar el shock. Estos niños requieren un acceso venoso rápido, y después del primer bolo, un bolo de $10 \mathrm{ml} / \mathrm{kg}$ debe repetirse si hay signos de persistir el shock. Tales niños también pueden necesitar apoyo general adicional incluyendo oxígeno y deben estar con monitoreos continuos y muy intensivos (Elliott, 2007).

Las historias clínicas deben incluir información sobre la duración y frecuencia de la diarrea, presencia de sangre o mucosidad en las heces, vómitos y si está manchada de bilis, uso y mezcla de fluidos de rehidratación en el hogar, así como otros aspectos habituales de la pediatría incluyendo enfermedades pasadas, estado de inmunización, alimentación, medicamentos y efectos secundarios relacionados. La Gestión Integrada de Enfermedad infantil (IMCI) ha simplificado la clasificación de la deshidratación en:

- No hay signos de deshidratación,

- Algo de deshidratación (en correlación con la antigua clasificación del 5\% de deshidratación)

- Deshidratación severa (correlacionando con la antigua clasificación del $10 \%$ de deshidratación).

Una forma relativamente objetiva de evaluar la deshidratación es comparando peso actual con un peso registrado recientemente, para calcular el porcentaje de deshidratación, tiempo de llenado capilar, turgencia de la piel y un patrón respiratorio anormal indicativo de acidosis se ha encontrado por revisión sistemática para ser los mejores indicadores de grado de deshidratación (Elliott, 2007). 


\section{Deshidratación en niños: Causas, diagnóstico y tratamiento}

Vol. 3, núm. 1., (2019)

Daniel Fernando Dick Paredes; Jenniffer Cristina Pazmiño Farfán; John Michael Franco Orellana; Adrián André Bravo Legarda

\section{Rehidratación y manejo de líquidos}

La observación de que el co-transporte de glucosa-sodio no se vio afectado en cólera y reconocimiento de que los procesos secretorios y de absorción en los intestinos están separados, lo que lleva a la formulación de la rehidratación oral. Solución (SRO), con concentraciones similares de sodio y glucosa que optimizan la absorción junto con potasio, cloruro y bicarbonato (Fonseca, 2004). Es considerado una de las innovaciones médicas más importante de los tiempos modernos y su promoción activa como parte del programa de la OMS para el control de las enfermedades diarreicas en la década de 1980. La mitad de las muertes por diarrea más de 20 años después de su implementación.

Una revisión Cochrane ha documentado que la rehidratación oral debería ser utilizada como primera línea para el manejo de niños con Gastroenteritis y algo de deshidratación. Cuando esto no es factible, la rehidratación enteral por vía nasogástrica es igual de eficaz, si no mejor, a la rehidratación intravenosa, con menos eventos adversos importantes y estancia hospitalaria más corta. Además, es más barato en términos de consumibles y recursos humanos y menos traumáticos para el niño.

La OMS, Sociedad Europea de Gastroenterología Pediátrica Hepatología y nutrición (ESPGHAN) y la Academia Americana de La pediatría (AAP) recomienda rehidratación rápida durante cuatro horas en deshidratación leve a moderada. La rehidratación debe ser más lenta de 8 a 24 horas en niños menores de tres meses de edad, aquellos con enfermedad respiratoria o cardíaca, aquellos con sospecha o probada Hipernatremia y en niños desnutridos. Ha habido una continua controversia sobre la composición ideal de ORS. Las preparaciones iniciales de la OMS 


\section{Deshidratación en niños: Causas, diagnóstico y tratamiento}

Vol. 3, núm. 1., (2019)

Daniel Fernando Dick Paredes; Jenniffer Cristina Pazmiño Farfán; John Michael Franco

Orellana; Adrián André Bravo Legarda

tuvieron mayor concentración de sodio debido a su desarrollo en áreas con alta incidencia de cólera (que se complica por la hiponatremia). Después de múltiples modificaciones las directrices actuales de la OMS recomiendan una SRO de osmolaridad reducida con concentraciones más bajas de sodio $(75 \mathrm{mmol} / 1$ vs $90 \mathrm{mmol} / \mathrm{l})$ y glucosa $(75 \mathrm{mmol} / 1$ vs 111 mmol /). Un metaanálisis ha demostrado su seguridad y eficacia tanto en el cólera como en la diarrea no cólera, con un menor uso de rescate de líquidos por vía intravenosa, vómitos reducidos y tasas similares de la hiponatremia cuando se reduce la osmolaridad y se compara con la estándar ORS (Guarino \& Albano, 2008) ESPGHAN, de hecho, recomienda una concentración aún más baja de sodio de $60 \mathrm{mmol} / 1$ para uso en niños con diarrea en Europa debido a la diferente etiología de la diarrea en entornos europeos.

Múltiples otras formulaciones modificadas de SRO también han sido ampliamente estudiadas. La SRO a base de arroz se puede utilizar como una terapia alternativa para SRO estándar en el cólera, ya que agrega un sustrato adicional de lumen al intestino sin aumentar la osmolalidad, proporcionando así moléculas de glucosa para la absorción mediada por glucosa. Sin embargo, no hay beneficio adicional en niños con diarrea sin cólera. Otras modificaciones incluyen almidón resistente a la amilasa que contiene SRO, ya que se postula que los carbohidratos no absorbidos aumentan la cadena de disponibilidad de ácidos grasos que mejoran la absorción colónica de sodio y agua, pero se necesitan ensayos adicionales para demostrar superioridad. La SRO también se ha combinado con goma guar, una mezcla de carbohidratos no digeribles, así como con probióticos, zinc y glutamina, pero actualmente no hay pruebas suficientes para ninguno de sus uso teniendo en cuenta las consideraciones adicionales de mayor costo, inestabilidad y disponibilidad de compuestos adicionales. 


\section{Deshidratación en niños: Causas, diagnóstico y tratamiento}

Vol. 3, núm. 1., (2019)

Daniel Fernando Dick Paredes; Jenniffer Cristina Pazmiño Farfán; John Michael Franco Orellana; Adrián André Bravo Legarda

\section{Mantener la nutrición}

Consenso de la OMS, ESPGHAN y AAP basado en el nivel 1 la evidencia es continuar amamantando en todo momento, y continuar la alimentación normal en gastroenteritis no complicada en cuatro horas. No hay papel para la dilución o reintroducción gradual de la fórmula o para fórmulas especiales como a base de soja o sin lactosa. No debe utilizarse bebidas con alto contenido de azúcar. Una comida extra al día por al menos una semana después de un episodio de gastroenteritis también se anima para permitir un crecimiento alcista (Bellemare. S, 2006).

\section{Terapia farmacológica}

La deficiencia de zinc es común en niños pequeños y se asocia con electrolito deteriorado y absorción de agua, disminución de la actividad de la enzima del borde del cepillo y deterioro celular y la inmunidad humoral. Los meta análisis han confirmado que la suplementación de zinc reduce la duración y la severidad de la diarrea persistente y reduce el riesgo de un episodio recurrente en los próximos 2-3 meses. OMS y UNICEF recomiendan suplementación de zinc (10 mg en niños menores de seis meses y 20 mg en niños mayores de seis meses) de 10 a 14 días como tratamiento universal. ESPGHAN sin embargo, no lo recomienda como una modalidad de tratamiento de rutina en niños europeos, a menos que estén desnutridos.

Los probióticos pueden ser un complemento eficaz del manejo de diarrea, pero es importante prescribir a los que han sido documentados para ser eficaz. Lactobacillus GG y Saccharomyces boulardii tiene los datos de eficacia más consistentemente documentados. Los mejores resultados se han demostrado en niños pequeños con virus gastroenteritis cuando el probiótico se administra temprano en el curso de la diarrea, a una dosis de $10^{10}$ UFC / día. Las 


\section{Deshidratación en niños: Causas, diagnóstico y tratamiento}

Vol. 3, núm. 1., (2019)

Daniel Fernando Dick Paredes; Jenniffer Cristina Pazmiño Farfán; John Michael Franco

Orellana; Adrián André Bravo Legarda

ventajas incluyen la reducción de la duración de la diarrea en un día reduciendo así costos de hospitalización, reducción de la diarrea persistente y reducción Spead a través de derramamiento viral. Hay preocupaciones sin embargo en países en desarrollo con respecto al uso de probióticos en el manejo de diarrea debido a la alta prevalencia de diarrea bacteriana en tales entornos clínicos, en los cuales las probióticas pueden ser menos eficaces y pueden surgir problemas de seguridad relacionados con la inmunosupresión.

El manejo de la diarrea por lo general es de apoyo no farmacológico. Agentes antimotilidad como la loperamida han sido asociada con enfermedad prolongada en la shigelosis, megacolon tóxico en Infección por C. difficile y síndrome hemolítico urémico en niños con la toxina Shiga que produce E. coli y no se recomiendan (Elliott, 2007).

Los antieméticos más antiguos tampoco se recomiendan debido al riesgo de efectos secundarios extrapiramidales, pero algunos agentes más nuevos como ondansetrón son efectivo sin efectos secundarios (Guarino \& Albano, 2008). No hay evidencia para apoyar el uso de prebióticos, glutamina, ácido fólico, caolín-pectina, atapulgita, carbón activado o bismuto. Sin embargo, puede haber potencial beneficio en el uso de esmectita, un silicato de aluminumagnesio que se une a la mucosa digestiva. La vitamina A no influye en el curso de la diarrea aguda, pero debe administrarse de acuerdo con las pautas para su efecto en la reducción de la mortalidad global.

\section{Manejo de niños con diarrea crónica}

En cuanto al niño que presenta diarrea aguda, debe haber una historia completa, incluida la historia familiar, y la evaluación clínica integral incluye el efecto sobre el estado nutricional de 


\section{Deshidratación en niños: Causas, diagnóstico y tratamiento}

Vol. 3, núm. 1., (2019)

Daniel Fernando Dick Paredes; Jenniffer Cristina Pazmiño Farfán; John Michael Franco Orellana; Adrián André Bravo Legarda

las personas con enfermedades de diarrea crónica. Es importante diferenciar si se trata de una diarrea persistente después de un episodio diarreico deshidratante agudo, como las causas pueden incluir sobrecrecimiento bacteriano en el intestino delgado, deficiencia de disacaridasa, desconjugación y deshidroxilación de sales biliares que inducen diarrea, sensibilización a proteínas, es decir, configuraciones clinicas que pueden requerir tratamiento especializado adicional.

El cuadro clínico del niño también es importante ya que puede ser indicativo de la causa de la diarrea, como el clásico de Toddler, diarrea en un niño próspero y deposiciones que contienen alimentos no digeridos y empeorando a medida que avanza el día. La necesidad de cualquier especialista, la investigación y el tratamiento dependerán del cuadro clínico del paciente y usualmente incluiría heces MC\&S para excluir parásitos, sustancias reductoras de heces y elastasa, y posiblemente prueba de sudor o serología celiaca (Rodríguez Soriano, 2000)

\section{Prevención}

En el momento del alta de un niño con un episodio de diarrea, el consejo debe ser en medidas para tratar de prevenir otro episodio y gestión del hogar apropiado para evitar la deshidratación. El Control de Enfermedades Diarreicas Mejoradas de la OMS (EDDC) se centra en una combinación de temas de salud pública como lavado de manos, preparación y almacenamiento de alimentos, así como agua potable y saneamiento; promoción de la lactancia materna (que ofrece una protección de seis veces); suplementación de zinc y vacunas contra el rotavirus. Se ha mencionado la enorme carga de la enfermedad por rotavirus y se cree que la introducción de la vacuna en los países en desarrollo tiene el potencial de ahorrar 600.000 vidas 


\section{Deshidratación en niños: Causas, diagnóstico y tratamiento}

Vol. 3, núm. 1., (2019)

Daniel Fernando Dick Paredes; Jenniffer Cristina Pazmiño Farfán; John Michael Franco

Orellana; Adrián André Bravo Legarda

de niños por año y ayuda para el logro del objetivo de desarrollo del milenio de reducir la mortalidad infantil en 2/3 para 2019.

\section{Conclusiones.}

Los resultados de la presente revisión confirman la importancia de algunas estrategias preventivas claves como lactancia materna, mejoras en el suministro de agua y saneamiento así como refinar algunos otros tales como la promoción de la higiene personal y doméstica, educación al destete / higiene de los alimentos. En los próximos años, la eficacia y la rentabilidad de muchos de estos métodos preventivos y las estrategias mejorarán, ya que las intervenciones no relacionadas con la vacunación implican cambios de comportamiento y salud pública. La educación para lograr esto será más efectiva. No hay un programa de control de enfermedades diarreicas, es probable que la implementación de todas las posibles estrategias preventivas, depende de una serie de factores tales como diferentes etiologías, infraestructura existente, y prioridades de los gobiernos. La implementación efectiva de estas estrategias preventivas requiere la participación de una variedad de sectores (agricultura, agua y suministro y saneamiento), más que para la gestión de casos clínicos.

\section{Bibliografía.}

Bellemare. S. (2006). Hartling L et al. Rehidratación oral versus terapia intravenosa para tratar la deshidratación debida a gastroenteritis en niños: un metanálisis de ensayos controlados aleatorios. The Cochrane Library.

Bolívar, J. (2015). Investigación Documental. México. Pax.

Burden of Disease report, M. R. (27 de Enero de 2010). http://www.mrc.ac.za/policybriefs/childmortality.pdf Accessed. 


\section{Deshidratación en niños: Causas, diagnóstico y tratamiento}

Vol. 3, núm. 1., (2019)

Daniel Fernando Dick Paredes; Jenniffer Cristina Pazmiño Farfán; John Michael Franco Orellana; Adrián André Bravo Legarda

Castro, J. (2016). Técnicas Documentales. México. Limusa.

Davila, A. (2015). Diccionario de Términos Científicos. Caracas: Oasis.

Elliott, E. (2007). Acute Gastroenteritis in Children. BMJ.

Fonseca, B. (2004). Terapia de rehidratación enteral versus intravenosa para niños con gastroenteritis: un metaanálisis de ensayos controlados aleatorios. Arch Pediatr Adolesc Med, 483-490.

Forsberg, B., \& Petzold, M. (2007). Diarrhoea case management in low-and middle-income countries -an unfinished agenda. Bulletin of the WHO.

Guarino, A., \& Albano, F. (2008). Evidence-based Guidelines for the Management of Acute Gastroenteritis in Children in Europe. European Society for Paediatric Gastroenetrology, hepatology and Nutrition/European Society for paediatric Infectious Diseases, (págs. 46:S81-S122.).

Naghipour, M., Nakgomi, T., \& Nakagomi, O. (2008). Issues with reducing the rotavirusassociated mortality by vaccination in developing countries. 6:3236-41.

Rodríguez Soriano, J. (2000). Fisiología de líquidos y electrolitos. Barcelona: Prous Science SA. 